\title{
Review
}

\section{Mobilizing artificial intelligence to cardiac telerehabilitation}

\author{
Jin $\mathrm{Su}^{1, \dagger}$, Ye Zhang ${ }^{1, \dagger}$, Qi-qi $\mathrm{Ke}^{1}$, Ju-kun $\mathrm{Su}^{1}$, Qiao-hong Yang ${ }^{1, *}$ \\ ${ }^{1}$ School of Nursing, Jinan University, 510632 Guangzhou, Guangdong, China \\ *Correspondence: yqiaohong@163.com (Qiao-hong Yang) \\ $\dagger$ These authors contributed equally. \\ Academic Editors: Brian Tomlinson and Takatoshi Kasai \\ Submitted: 26 November 2021 Revised: 3 January 2022 Accepted: 12 January 2022 Published: 25 January 2022
}

\begin{abstract}
Cardiac telerehabilitation is a method that uses digital technologies to deliver cardiac rehabilitation from a distance. It has been shown to have benefits to improve patients' disease outcomes and quality of life, and further reduce readmission and adverse cardiac events. The outbreak of the coronavirus pandemic has brought considerable new challenges to cardiac rehabilitation, which foster cardiac telerehabilitation to be broadly applied. This transformation is associated with some difficulties that urgently need some innovations to search for the right path. Artificial intelligence, which has a high level of data mining and interpretation, may provide a potential solution. This review evaluates the current application and limitations of artificial intelligence in cardiac telerehabilitation and offers prospects.
\end{abstract}

Keywords: Cardiac rehabilitation; Artificial intelligence; Telerehabilitation

\section{Introduction}

As the leading cause of worldwide deaths, cardiovascular diseases caused an estimated 18.6 million deaths in 2019 [1]. According to the World Health Organization (WHO), cardiovascular diseases represent $38 \%$ of premature deaths (age $<70$ years) due to noncommunicable diseases [2]. At present, the number of patients living with cardiovascular diseases is still considerable. In data up to 2017, an estimated 108.7 million people were living with cardiovascular diseases in the 54 member countries of the European Society of Cardiology [3]. The condition is quite worse. Based on the 2018 data from the Medical Expenditure Panel Survey (MEPS), the annual total expenditures of heart diseases in the United States, including direct and indirect costs, is an estimated $\$ 108.56$ billion [4]. Treatment costs and productivity loss because of premature deaths have brought a heavy economic burden to the global health care system [1]. One essential method to ensure favorable clinical outcomes and increase the quality of life in patients with cardiovascular diseases, thereby releasing the burden of disease treatment, is implementing cardiac rehabilitation (CR) [5].

Back in 1995, CR was defined as "the provision of comprehensive long-term services involving medical evaluation, prescriptive exercise, cardiac risk factor modification, education, counseling, and behavioral interventions" [6]. This definition was then updated to one with wider connotations, containing specific core components to optimize cardiovascular risk reduction, foster healthy behaviors and compliance to these behaviors, reduce disability, and promote an active lifestyle for patients with cardiovascular diseases [7].
The safety and efficacy of CR have been well explored by previous studies. CR has been found effective in improving cardiac functions, reducing diseases recurrence, hospital readmission, and mortality in patients with cardiovascular diseases, and is cost-effective [8-13]. CR has been recognized as a class $1 \mathrm{~A}$ recommendation for secondary prevention of cardiovascular diseases by the European Society of Cardiology, the American Heart Association, and the American College of Cardiology Foundation [14,15].

Unfortunately, there are still some problems in the delivery of CR. The problem of low participation and completion among eligible patients remains largely tricky. Between 2007 and 2011, participation in CR was lower than $20 \%$ in both Medicare sampling and the Veterans Affairs (VA) healthcare system in the United States [16]. Although with the improvement of public awareness about health, this situation has improved, it is still suboptimal. The National Audit of Cardiac Rehabilitation (NACR) reported that only $50 \%$ of eligible patients on average were accepting CR across England, Northern Ireland, and Wales in 2018 [17]. Moreover, the imbalanced distribution of healthcare resources also needs to be considered; this imbalance is likely to lead to the unavailability of CR for patients in some low- and middle-income countries. According to research in 2019 , CR is available in only $54.7 \%$ of countries globally, and this rate is low to $17.0 \%$ in Africa [18]. The ongoing pandemic caused by COVID-19, a novel coronavirus called coronavirus disease-2019, has brought new challenges to CR programs [19]. The lockdowns of cities or countries, maintaining social distance, and quarantine at home have all further hampered patients' adherence to CR programs. Moreover, many CR centers were closed to prevent crossinfection among outpatients. 
Cardiac telerehabilitation may be a feasible solution [20]. Using innovative information and communication technologies to deliver CR from a distance is called telerehabilitation or home-based CR, which was previously used as a partial alternative to center-based CR [21]. Noninferiority or superiority of cardiac telerehabilitation has been shown compared to center-based CR. Specifically, cardiac telerehabilitation could successfully improve patient activation and health literacy, further improving adherence and completion of rehabilitation [22,23]. Moreover, cardiac telerehabilitation has been shown to be more helpful than center-based CR for patients to build a healthy lifestyle, such as weight reduction and smoking cessation [23,24]. Some studies have concluded that cardiac telerehabilitation could enhance cardiorespiratory fitness and quality of life $[23,25,26]$, and lower the rate of readmission or major adverse cardiac events [24,27]. In addition, cardiac telerehabilitation provides flexibility to patients in terms of arranging rehabilitation time and location, which may satisfy their preference for rehabilitation settings and improve their willingness to participate in CR $[28,29]$. Although there is still no strong evidence to prove that telerehabilitation benefits participants [30], it is worth promoting, because it means a lot to face the coronavirus challenge and to ease the unfair distribution of $\mathrm{CR}$ resources. After the outbreak of the pandemic, the European Association of Preventive Cardiology (EAPC) published a structured call-for-action that suggested maintaining continuity in the delivery of CR through comprehensive telerehabilitation [30]. Furthermore, with the growing use of cardiac telerehabilitation, difficulties during implementation have arisen. The general digital technologies used in telerehabilitation, including smart support systems and wearable monitoring devices, ensure convenient remote monitoring and data collection, while also bringing considerations for clinicians about how to analyze and manage these "big data" effectively. Some innovations need to be implemented to respond to previous and emerging problems.

Mobilizing artificial intelligence (AI) into cardiac telerehabilitation may provide a potential path to improve uptake and delivery. The number of studies investigating cardiac telerehabilitation has gradually grown since the pandemic. Most studies combine AI algorithms with digital health devices during the implementation of cardiac telerehabilitation, but few reviews have summarized the functions of AI algorithms in these combinations which are necessary to help the public to understand these technologies. Among the existing reviews, the short-term effects of AI in cardiac telerehabilitation have gained attention. However, few have touched on the potential application of AI in long-term cardiac telerehabilitation, which we have tried to address in this review. Hence, this review is based on two commonly used digital devices, wearable monitoring and support systems, to evaluate the application of AI in cardiac telerehabilitation. The review highlights the four primary functions of combining AI algorithms in cardiac telerehabilitation to reach a better interpretation of these novel technologies, providing references for future delivery of cardiac telerehabilitation during the pandemic, as well as pointing out some potential ethical and legal problems that need to be addressed in attended by future research.

\section{Artificial intelligence in cardiology}

$\mathrm{AI}$ is a field of computer science that aims to mimic human thought processes, learning capacity, and knowledge storage [31]. There are two main subfields of AI: various types of machine learning (ML) and cognitive computing [31]. Fig. 1 shows the composition of AI. Nowadays, AI has been broadly studied in fields such as engineering [32,33], medicine [34,35], psychology [36], and economics [37]. Furthermore, AI-based technologies are integrated into our daily life, for example in the forms of object and speech recognition [38-40] and product recommendation [41]. During the COVID-19 pandemic, the possibility of AI to be an effective tool for healthcare systems has also been explored by several research studies and showed that AI has improved diagnosis and treatment, contact tracing, drug/vaccine development [42-46]. Similarly, it is not novel to mobilize AI algorithms in cardiology. AI is helpful for clinicians to exploit big data and implement precision cardiovascular medicine. The benefits of applying different AI algorithms in cardiology have been confirmed in previous research. Using a deep neural network to accurately classify arrhythmias from electrocardiogram (ECG) data could reduce misdiagnosis resulting from computerized ECG interpretations [47]. An artificial neural nets (ANN) model could achieve higher accuracy in the early prediction of non-ST-elevation myocardial infarction patients with chest pain, providing valuable insight in clinical diagnosis [48]. A supervised machine learning model could be used for survival prediction, which would help to determine the mechanisms of right ventricular failure in pulmonary hypertension [49]. These AI algorithms or models conform to the needs of highly efficient and personalized, widely accessible cardiac telerehabilitation.

\section{Wearable monitoring with artificial intelligence}

Wearable monitoring is based on wearable sensors that are usually worn as a wristband or embedded in a smartwatch or mobile phone [50]. These technologies release cardiac telerehabilitation from the constraints of time and location-limits, collecting more comprehensive and objective data in free-living conditions which may provide some new insights for researchers [51]. However, the challenges are how to assess and properly interpret cardiac telerehabilitation progression based on the massive amounts of data collected [52]. Furthermore, the quality and relevance of the data gathered is a matter, because the data collected by wearable sensors might include distracting and unusable 


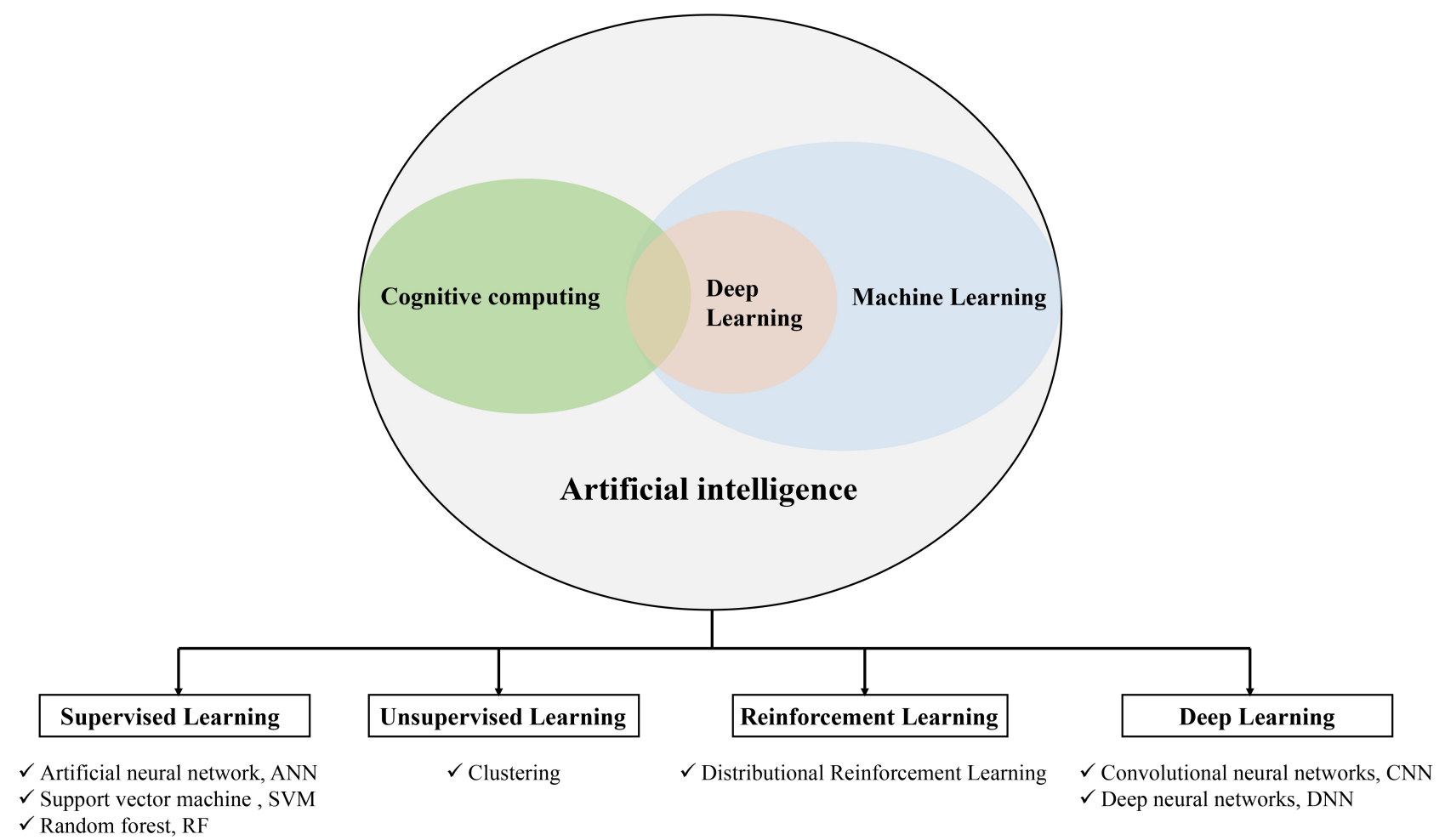

Fig. 1. Common AI algorithms. Machine learning is a subdiscipline of AI, which has become the chief AI tool. AI uses the concept of learning and can be divided into supervised learning, unsupervised learning, reinforcement learning, and deep learning.

data [53]. Limited battery capacity might be one of the reasons for the suboptimal data [54,55]. Also, the comfort in attaching and wearing the wearables has been evaluated by patients as an essential factor in their acceptance of wearables [56]. Any discomfort due to wearable devices might aggravate the premature termination of exercises, thus lowering data completeness. Although the difficulties caused by low-quality data are mainly associated with the limitations of wearable devices, an efficient and effective datahandling method could be helpful for solving this problem. Accurately identifying and correctly interpreting the data and uncovering potential patterns in the dataset with AI would support cardiac telerehabilitation in meeting the challenges posed [57].

\subsection{Fitness detection and recognition}

Wearables enable continuous ambulatory fitness monitoring of patients [58]. Identifying various activities of the human body in real time needs an efficient and wellworking model which may involve the application of some AI algorithms. Previous studies have shown the feasibility of using machine learning to design and train an accurate classifier for data feature selection, which enables wearables to capture and recognize various kinds of human activities during cardiac telerehabilitation [59,60]. Fig. 2 shows the main steps of the ML classification algorithm. The reliability of the support vector machine (SVM) classi- fier, one kind of ML classifier, was tested according to the process of Leave-one-subject-out cross validation, resulting in an accuracy rate of $95.4 \%$ in classification [59]. In this study, individual features of patients ranked in the top ten were selected to train the SVM model [59]. Features selection is the most crucial aspect of building a highly accurate classifier. One research used a method of classifier verification called 12-fold cross validation in the process of ML model and achieved an accuracy of $97 \%$ in the classification of physical activity [60]. The most error was generated from upstairs/downstairs being classified as walking, which indicates AI models are limited in identifying actions in the same genre [60]. Another research study used a different kind of AI model, the convolutional neural network (CNN) model, and showed higher accuracy in exercise recognition, compared to the traditional approach (supervised ML) [61]. In exercise recognition, the SVM model was found to be the best performing supervised ML model with an overall accuracy measure of $96.07 \%$, while the deep CNN model achieved a higher one of $96.89 \%$ [61]. This research used wearable sensors to collect signals of twelve limb movements in rehabilitation and form datasets to train, test, and validate models [61]. Training models to recognize basic limb movements could be the potential solution for AI models to distinguish similar body actions in cardiac telerehabilitation. In addition, different kinds of AI models may perform at different levels. Comparisons among algorithms 


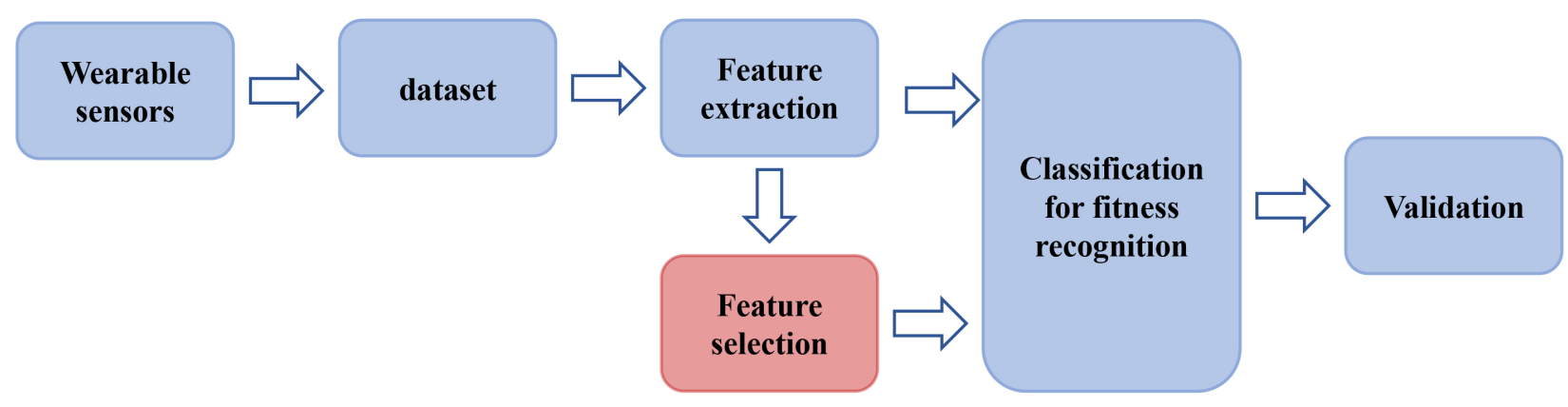

Fig. 2. Main steps of ML classification algorithm. The ML classification algorithm consists of the following steps: transforming collected signals to form a dataset, extracting features from data, feature selection/feature reduction, classification by algorithms, validating results.

Table 1. AI model development for fitness detection and recognition.

\begin{tabular}{|c|c|c|}
\hline Algorithm & Model function & Description \\
\hline SVM & Physical activity classification. & $\begin{array}{l}\text { Features: top ten features in time-domain and frequency domain [59]. } \\
\text { Feature selection method: Relief-F algorithms [59]. }\end{array}$ \\
\hline CSVM & Physical activity classification. & $\begin{array}{l}\text { Features: seven features were selected finally: co-relation } \mathrm{X} \text { and } \mathrm{Y} \text { axis, co- } \\
\text { relation } \mathrm{Y} \text { and } \mathrm{Z} \text { axis, minimum value along } \mathrm{X} \text { axis, kurtosis of data around } \mathrm{X} \\
\text { axis, skewness around } \mathrm{X} \text { axis, standard deviation } \mathrm{X} \text { axis, the sum of aggregate } \\
\text { acceleration } \mathrm{Ai} \text { (if } \mathrm{Ai} \leq 25 \text { th percentile) [60]. } \\
\text { Feature selection method: } 12 \text {-fold cross validation. Combining feature selection } \\
\text { with model validation. Whether the feature was selected or not was decided by } \\
\text { the accuracy of the retrained model after removing the feature [60]. }\end{array}$ \\
\hline $\mathrm{CNN}$ & Fitness recognition. & $\begin{array}{l}\text { Using data collected by 3D accelerometers and 3D gyroscopes to build the LME } \\
\text { exercise datasets (INSIGHT-LME dataset) include training sets, validation sets, } \\
\text { and test sets, to develop models [61]. }\end{array}$ \\
\hline
\end{tabular}

Abbreviations: SVM, support vector machine; CNN, convolutional neural network.

and methods of feature selection are important to find the optimal AI model of cardiac telerehabilitation, which is a long tedious process and needed further research. Table 1 (Ref. [59-61]) lists the different features chosen to train models and the feature selection method used in these studies. Extending beyond fitness detection and recognition, an AI server was used to identify and manage abnormal vital signs when patients were doing physical activities of CR at home [62]. These design approaches implement a clear and correct interpretation of data about physical activities and allow clinicians to assess patients' performance in physical activities, offering objective evidence for updating cardiac telerehabilitation exercise plans.

\subsection{Tracking and interpreting cardiac functional capacity}

Further, AI has been used with wearables to assess and track the prognosis of cardiac telerehabilitation. Wearables with an accelerometer and electrocardiogram could obtain heart rate parameters as well as estimate patients' effort during walking, which could function as the input data for machine learning algorithms to predict the 6-minute walk test distance [52]. Compared to the actual 6-minute walk test distance, the AI prediction model in the referenced study made an overestimation of $42.8 \mathrm{~m}$ ( \pm 36.8 m) [52], which is below $50 \mathrm{~m}$ and could be considered as non-significant difference according to the clinical threshold for detecting changes in disease state [63]. A previous study showed a mean difference of $2 \mathrm{~m}( \pm 7.84 \mathrm{~m})$ between the predicted and actual 6-minute walk distance in patients with cardiopulmonary conditions [64]. This model showed a better function in prediction accuracy. Hence, the algorithm-based prediction model of functional capacity in cardiac telerehabilitation might need advanced adjustments to improve its accuracy and reliability. However, these studies nevertheless show the possibility of objectively tracking and interpreting cardiac functional capacity during cardiac telerehabilitation by combining AI with wearable monitoring, which might enlighten further studies. It should be mentioned that because of the significant variability of physiological signals, the wearables collected, 
and the complicated subgroup component of cardiovascular diseases, it might be very hard to be model based on limited datasets [65]. In future studies, more related physiological features such as respiration or $\mathrm{SpO}_{2}$ could be added to refine the models [52]. In addition, evaluating whether such models have the same efficiency in a subgroup population of cardiovascular diseases should be investigated [52].

Increased public attention should be devoted to identifying an effective method to follow-up patients' condition with cardiac telerehabilitation during the COVID-19 pandemic. The benefits of CR in long-term clinical outcomes have been proven and are well known [66]. Some studies have explored the long-term effects of cardiac telerehabilitation and concluded satisfactory results in some health outcomes such as cardiorespiratory fitness [67] and physical activities [68,69]. However, studies are still lacking and more data are needed to determine the long-term benefits of cardiac telerehabilitation [70]. Except for cardiac functional capacity, other long-term effects such as patients' exercise adherence in cardiac telerehabilitation should also be paid attention to. Recent research has shown that there is no significant difference in exercise training intensity between patients who participated in cardiac telerehabilitation versus standard CR [71]. However, some existing research studies have concluded that the low usage rates of wearable devices in the long-term follow-up of telerehabilitation could reflect a low adherence to using wearables [55,72]. To address the inconsistent views about the patients' exercise adherence in cardiac telerehabilitation, further research is necessary. Therefore, more comprehensive tracking and interpreting are necessary for long-term follow-up of cardiac telerehabilitation [73]. As the special period with the coronavirus disease will probably continue for a long period [74], it is increasingly important to explore AI more in the longitudinal follow-up of cardiac telerehabilitation.

\section{Support systems with artificial intelligence}

During cardiac telerehabilitation, communication between patients and healthcare workers is conducted remotely by using the telephone, internet, and video conferencing [21]. In early telerehabilitation, consultations and education were predominantly delivered by phone call [7577], which limited the rehabilitation services to be provided. Technological innovation has brought rapid growth in the use of the internet and mobile phones, particularly smartphones [78]. This revolution in information communication has enabled more diverse delivery methods of telerehabilitation [24]. For instance, automated text messages have been used to provide suggestions, motivational reminders for lifestyle modification, and adherence improvement $[79,80]$. Based on the internet, secure websites have been utilized for cardiac telerehabilitation. Scheduled sessions for education or one-to-one consulting with rehabilitation specialists could be achieved in web-based interventions, and patients could communicate with professionals by e-mail if they have any questions outside sessions [81]. Other chatting methods such as a form similar to e-mail but within a website [82] could also ensure timely communication between patients and rehabilitation teams. With the development of mobile health and remote monitoring technology, smart systems for disease management have been generally used. A comprehensive support system of cardiac telerehabilitation allows the generation and saving of medical records which enable clinicians to objectively supervise and follow up the changes in patients' situations dynamically [83]. Online platforms connect patients and clinicians over a long distance, enabling patient consultations and health education, and prescription adjustment [23]. While comprehensive cardiac telerehabilitation sometimes needs to measure several indicators, it takes time for clinicians to learn about such amounts of information and devise patientspecific programs, resulting in a mistimed response to patients. AI may be a promising solution to address this difficulty.

\subsection{Triage for preliminary intervention}

Support systems could comprehensively monitor key measurements of CR [84,85]. AI algorithms provide an efficient method to assess and manage these measurements in real time. A HEARTEN Knowledge Management System (KMS) was designed to support heart failure patients, which uses machine learning for feature selection and classification of data, allowing automated stratification of rehabilitation risk, disease severity, and patients' adherence [86]. In this situation, the combination of AI algorithm could provide rating results about the observed indicators. Data were received from hospital records, biosensors, and sensors in this study, including 11 categories [86]. The Random Forest algorithm was employed for classification and the 10fold stratified cross validation approach was used for evaluations of results [86]. 95\% accuracy has been achieved by the diseases' severity module, $85 \%$ accuracy for medication adherence, and $78 \%$ accuracy for the overall adherence risk module [86]. Accordingly, timely preliminary interventions, such as professional suggestions or education knowledge to different health grades, could be provided by support systems. Similarly, in the text-based cardiovascular rehabilitation program, AI was used to review and triage text messages into two groups, depending on whether it was a complex situation requiring further review from a staff member or was simple enough to be solved by suggestions [87]. A low false-negative rate indicates few messages which need a response would be missed so that the research aimed to develop the model with the lowest false-negative rate [87]. Evaluating by a binary classification evaluator, the ensemble model (with all tested machine-learning models combined) achieved the lowest false negatives of $1.43 \%$ and $16.2 \%$ false positives, which means that health professionals would have to review about $36.9 \%$ (20.7\% true positives plus $16.2 \%$ false positives) of all the incoming text 
messages [87]. Although the false-negative rate was very low, the accuracy was moderate because the false positives still need to be lowered. AI model development information for automated stratification is described in Table 2 (Ref. [86,87]). Moreover, an AI-driven healthcare system was envisioned by researchers in optimal perspective, in which AI algorithms could automatically offer decision support on medication and physical activity prescription in most instances [88]. This envisioning provides a direction for future design.

Consequently, it could be said that AI helps to improve the feasibility of cardiac telerehabilitation systems, reducing the time cost and workload of healthcare staff. Feedback on the rehabilitation problems of patients with more targeted recommendations could also be provided within a shorter period. As shown in Table 2, support systems of cardiac telerehabilitation can manage amounts of data which allows more features to be selected to train and test AI models. However, the triage bias caused by AI models is a potential ethical issue that should be considered. Future work is required to validate the AI models in larger datasets if research results are going to be generalized to other subgroup populations [86,87]. Because the amount of available literature is limited, the effectiveness and safety of preliminary interventions by support systems with AI still need more applications to be certified.

\subsection{Identifying predictors for tailored cardiac rehabilitation}

AI has been used for a more accurate prediction of CR prognosis, owing to the strong data-mining function it has for deriving relationships and statistical inference from datasets [89,90]. Furthermore, important predictors for the participation and completion of CR using AI models have also been discovered by researchers [91]. This could promote the personalization of CR programs, which is necessary to promote wider utilization [92]. Although the present studies in this field did not combine the output AI model with support systems of cardiac telerehabilitation, it still could provide some meaningful perspectives for the development of tailored cardiac telerehabilitation programs. For that, further feasibility studies are necessary to ascertain the possibility of identifying predictors by applying AI models in cardiac telerehabilitation support systems.

\section{Current limitations of implementation}

\subsection{The possibility of greater inequalities}

Cardiac telerehabilitation would be able to provide more opportunities for eligible people who cannot reach the CR services in their local area. The utilization of AI can help telerehabilitation technology become more efficient and feasible. However, to broadly implement new technology in some fields, some economic and social gaps may need to be addressed. What cannot be ignored is that the digital health devices and AI algorithms carry the addi- tional challenge of digital literacy, which indicates the ability to appraise and apply information or knowledge gained from electronic sources [93]. Engaging innovative technologies like AI might lead to health services being unavailable for those patients with limited digital literacy. The greatest challenge would be how to maximize the benefits of AI to provide efficient and tailored telerehabilitation services, while avoiding worsening wealth and health inequalities and increasing unemployment [94]. Whether the combination of AI with cardiac telerehabilitation is more complex to implement among people in low-income countries, enabling them to acquire medical resources, still needs to be further researched.

\subsection{Ethical issues of artificial intelligence}

The use of AI in healthcare is always debatable, mainly with respect to data privacy and security, transparency and fairness of algorithmically automated decisions, algorithmic accountability, and liability [95]. National and international organizations and private enterprises have responded to the concerns by publishing guidelines or principles, which demonstrate the need for ethical AI [96].

\subsubsection{Data protection and data privacy}

The application of AI needs an amount of data for building up and training models, which may include personal information or clinical data of patients [97]. How to protect the security of patients' data urgently needs to be considered carefully to maintain the human rights of privacy. Data provenance and permission for use are particularly important. Adopting measures to prevent the unethical use of patients' data is necessary, such as ensuring the rights of patients to be informed, have access, and be allowed rectification [98]. The General Data Protection Regulation (GDPR) endorsed by the European Union aims to regulate and standardize personal data use, strengthening and unifying the data protection for all individuals within the European Union [99]. Specific informed consent requirements for using and granting data, and several rights that must be respected in data processing, were set up in this law [99]. Many published guidelines have discussed data protection and data privacy; however, further specification is essential because of the complexity and diversity of the datasets, including knowledge and cultural pluralism around the world [96]. Data protection guidelines or laws might need to be adjusted to fit the local situation in different countries or states, and data used in different conditions should be bound by different legal protections [100].

\subsubsection{Transparency and fairness}

Transparency and fairness of algorithms are the most prevalent issues mentioned. The highly automatic decisionmaking ability enables AI to make decisions without human intervention [95]. Indeed, this function brings more effi- 
Table 2. AI model development for automated stratification.

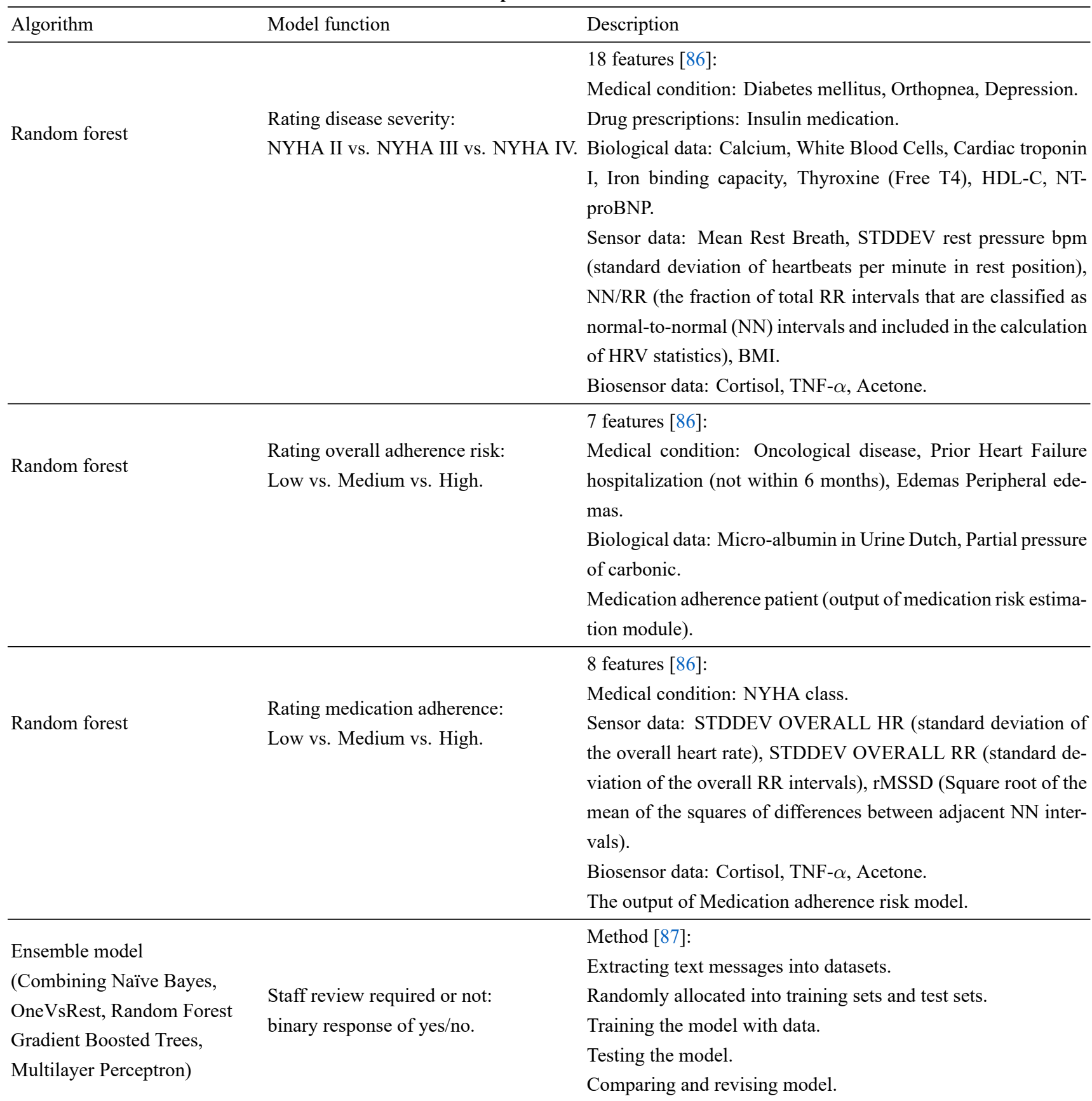

Abbreviations: NHYA, New York Heart Association; HDL, high-density lipoprotein; NT-proBNP, N-terminal pro-B-type natriuretic peptide hormone; TNF, tumor necrosis factor.

cient data management but also makes algorithms harder to explain. However, unmatured AI models might obtain results that reflect pre-existing bias in the real world if models were trained by unrepresentative or inadequate datasets $[100,101]$. This kind of algorithm bias could entrench or exacerbate health disparities [102]. Despite the notion of bias being very complex, and biases commonly existing in the human world, it is possible and ethically necessary to design AI systems to help offset human biases to try to lead to outcomes closer to fairness [103]. For now, to achieve greater transparency, it is suggested to improve the interpretability and auditability of AI [104]. Nonetheless, a contradiction could likely be involved between open-access data and algorithms and patients' privacy, which still needs future consideration. Recently, an article commented that bias in AI models is not a feature of data that could be simply eliminated but needs a fundamental realignment of the culture of software development to address, which should acknowledge that developers have necessary responsibilities for patient health and welfare [101]. 


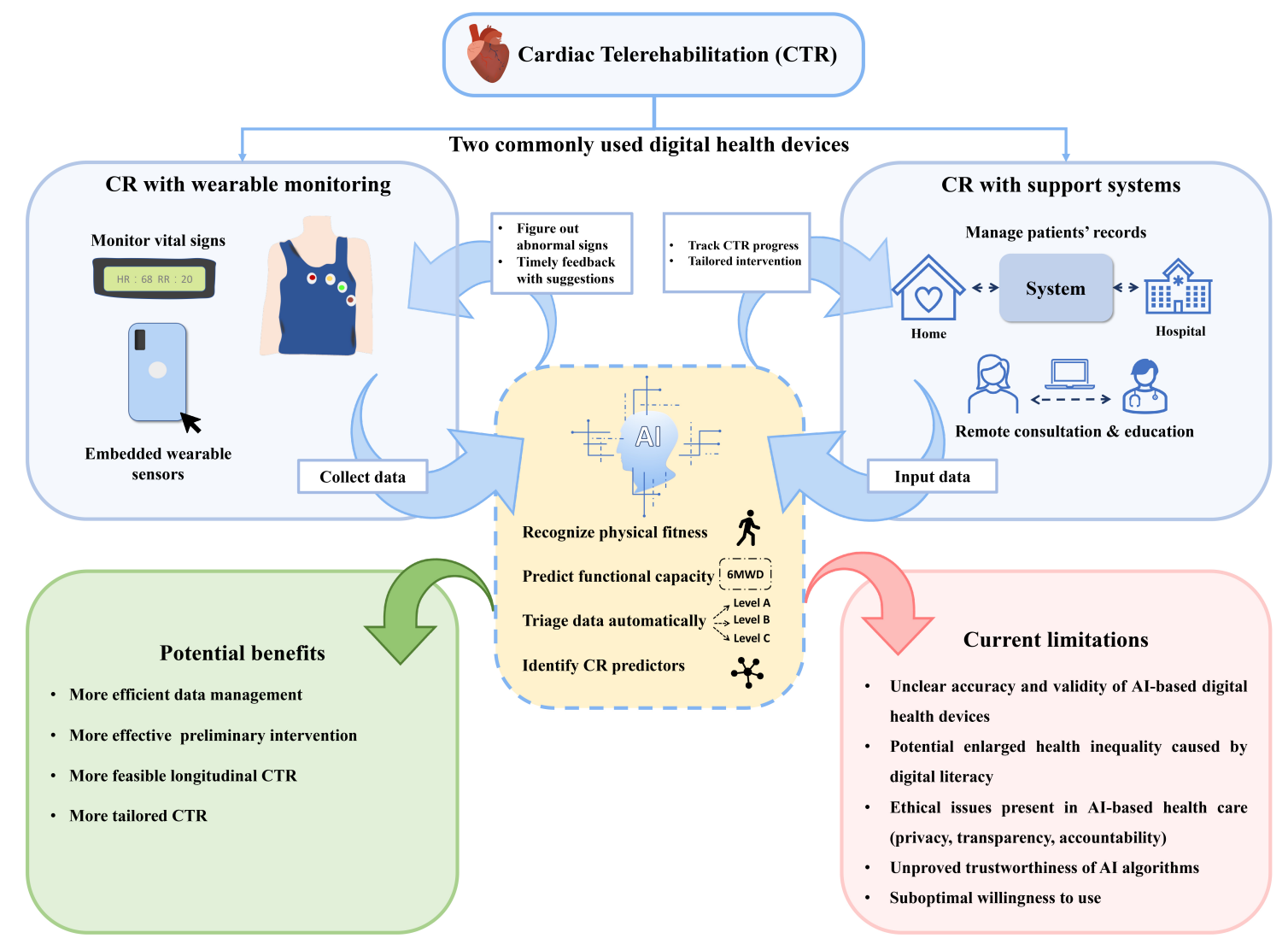

Fig. 3. Application of AI in Cardiac telerehabilitation. Wearable monitoring and support systems are two commonly used digital health devices. By combining them, AI can figure out abnormal signs and timely feedback with suggestions, also enabling health workers to track cardiac telerehabilitation progress and present tailored intervention. Although with such benefits, some limitations of AI-based cardiac telerehabilitation are still necessary to be considered.

\subsubsection{Responsibility and accountability}

Accountability is needed in AI algorithms to clarify who should be liable for decisions made with algorithmic support [105]. The accountability component involves multiple professionals, which is considered the most challenging part of implementation [102]. While responsible AI has garnered widespread attention, whether we should consider AI algorithms as a subject of responsibility or whether we should consider humans as the only actors who are ultimately responsible for algorithm-based decisions remains debatable [96]. Statements about the specific actors accountable for AI's decisions are also diverse [96]. In fact, the responsibility may be difficult to determine. Without transparency, it is hard to enforce accountability [95]. As discussed above, it is still difficult to achieve transparency. Moreover, it is still unclear whether algorithm-based mistakes are related to the quality of data input [95].

In conclusion, the ethical issues of AI remain sticky when handled and should be focused upon to be attentive to them and further reviewed to improve the regulatory system.

\subsection{Trustworthiness and willingness to use aspects}

Not all clinicians and patients are interested in adapting to new technologies. Some clinicians do not trust AI due to the difficulties associated with understanding how it works, which may affect their willingness to use AI-based products [106]. This situation seems more serious in patients. Previous research shows that only $20 \%$ of patients think there are more advantages than disadvantages in the application of AI, and $35 \%$ of participants would refuse to implement their disease-related intervention through AI [107]. Patients worry about losing connection with clinicians and are also concerned about the misuse of AI that can cause additional damage to their health, which may be caused by inadequate follow-up and insufficient education [108]. Achieving public trust is necessary to operate AI-based cardiac telerehabilitation [109]. Some opinions warn that people should be cautious about overtrusting algorithms [110], and one of the most critical things to do now is to boost the construction of trustworthy AI models with the cooperation of related organizations or enterprises [111]. To mobilize AI to cardiac telerehabilitation, it seems clear that we still have a lot to do. 


\section{Conclusions}

Tailored and ambulatory cardiac telerehabilitation can be achieved by applying AI into wearable monitoring and support systems. With AI algorithms or models, wearables could accurately detect and identify the physical activities of human beings during cardiac telerehabilitation to assess the cardiac function capacity of patients, which makes it possible for the longitudinal follow-up of cardiac telerehabilitation. AI combined with cardiac telerehabilitation support systems could analyze observed indicators in real time and then triage the results, which enables systems to provide timely feedback and more targeted recommendations to different grades as preliminary interventions. Moreover, based on the results, systems could automatically refer patients with poor results or complex situations to staff for further reviews.

Fig. 3 has concluded the application of AI in cardiac telerehabilitation. AI could improve the efficacy and effectiveness of cardiac telerehabilitation, helping to make it comprehensive and close to optimal. For now, further evidence is needed to assess the feasibility and safety of the implementation of cardiac telerehabilitation with AI in the clinic. Furthermore, the potential ethical and legal issues of applying AI should be sufficiently acknowledged by researchers. Further cooperation of multiple disciplines could be fostered to consider solutions to the current limitations.

\section{Author contributions}

JS and YZ wrote the manuscript with support from QHY. JS, YZ, QQK, and JKS revised the manuscript under the guidance of QHY. All authors read and approved the final manuscript.

\section{Ethics approval and consent to participate}

Not applicable.

\section{Acknowledgment}

We would like to thank all contributing authors and peer reviewers for their precious opinions and suggestions.

\section{Funding}

This research received no external funding.

\section{Conflict of interest}

The authors declare no conflict of interest.

\section{References}

[1] Virani SS, Alonso A, Aparicio HJ, Benjamin EJ, Bittencourt MS, Callaway CW, et al. Heart Disease and Stroke Statistics2021 Update: A Report From the American Heart Association. Circulation. 2021; 143: e254-e743.

[2] Cardiovascular diseases (CVDs). World Health Organization. 2021. Available at: https://www.who.int/news-room/fact-sheet s/detail/cardiovascular-diseases-(cvds) (Accessed: 15 November 2021).
[3] Timmis A, Townsend N, Gale CP, Torbica A, Lettino M, Petersen SE, et al. European Society of Cardiology: Cardiovascular Disease Statistics 2019. European Heart Journal. 2020; 41: $12-85$.

[4] Agency for Healthcare Research and Quality. Medical Expenditure Panel Survey. Total expenditures (\$) in millions by condition, United States, 2017 to 2018. 2018. Available at: https: //datatools.ahrq.gov/meps-hc (Accessed: 15 November 2021).

[5] Ambrosetti M, Abreu A, Corrà U, Davos CH, Hansen D, Frederix I, et al. Secondary prevention through comprehensive cardiovascular rehabilitation: from knowledge to implementation. 2020 update. a position paper from the Secondary Prevention and Rehabilitation Section of the European Association of Preventive Cardiology. European Journal of Preventive Cardiology. 2021; 28: 460-495.

[6] Wenger NK, Froelicher ES, Smith LK, Ades PA, Berra K, Blumenthal JA, et al. Cardiac rehabilitation as secondary prevention. Agency for Health Care Policy and Research and National Heart, Lung, and Blood Institute. Clinical Practice Guideline. Quick Reference Guide for Clinicians. 1995; 1-23.

[7] Balady GJ, Ades PA, Comoss P, Limacher M, Pina IL, Southard $\mathrm{D}$, et al. Core Components of Cardiac Rehabilitation/Secondary Prevention Programs. Circulation. 2000; 102: 1069-1073.

[8] Bellmann B, Lin T, Greissinger K, Rottner L, Rillig A, Zimmerling S. The Beneficial Effects of Cardiac Rehabilitation. Cardiology and Therapy. 2020; 9: 35-44.

[9] Chen Y, Wang C, Lai Y, Liao Y, Wen Y, Chang S, et al. Homebased cardiac rehabilitation improves quality of life, aerobic capacity, and readmission rates in patients with chronic heart failure. Medicine. 2018; 97: e9629.

[10] Dalal HM, Taylor RS, Jolly K, Davis RC, Doherty P, Miles J, et $a l$. The effects and costs of home-based rehabilitation for heart failure with reduced ejection fraction: The REACH-HF multicentre randomized controlled trial. European Journal of Preventive Cardiology. 2019; 26: 262-272.

[11] Peersen K, Munkhaugen J, Gullestad L, Liodden T, Moum T, Dammen $\mathrm{T}$, et al. The role of cardiac rehabilitation in secondary prevention after coronary events. European Journal of Preventive Cardiology. 2017; 24: 1360-1368.

[12] Shields GE, Wells A, Doherty P, Heagerty A, Buck D, Davies LM. Cost-effectiveness of cardiac rehabilitation: a systematic review. Heart. 2018; 104: 1403-1410.

[13] van Halewijn G, Deckers J, Tay HY, van Domburg R, Kotseva $\mathrm{K}$, Wood D. Lessons from contemporary trials of cardiovascular prevention and rehabilitation: a systematic review and metaanalysis. International Journal of Cardiology. 2017; 232: 294 303.

[14] Piepoli MF, Hoes AW, Agewall S, Albus C, Brotons C, Catapano AL, et al. 2016 European Guidelines on cardiovascular disease prevention in clinical practice: The Sixth Joint Task Force of the European Society of Cardiology and Other Societies on Cardiovascular Disease Prevention in Clinical Practice (constituted by representatives of 10 societies and by invited experts) Developed with the special contribution of the European Association for Cardiovascular Prevention \& Rehabilitation (EACPR). European Heart Journal. 2016; 37: 2315-2381.

[15] Smith SC, Benjamin EJ, Bonow RO, Braun LT, Creager MA, Franklin BA, et al. AHA/ACCF secondary prevention and risk reduction therapy for patients with coronary and other atherosclerotic vascular disease: 2011 update: a guideline from the American Heart Association and American College of Cardiology Foundation endorsed by the World Heart Federation and the Preventive Cardiovascular Nurses Association. Journal of the American College of Cardiology. 2011; 58: 2432-2446.

[16] Beatty AL, Truong M, Schopfer DW, Shen H, Bachmann JM, Whooley MA. Geographic Variation in Cardiac Rehabilitation 
Participation in Medicare and Veterans Affairs Populations Opportunity for Improvement. Circulation. 2018; 137: 1899-1908.

[17] British Heart Foundation (BHF). The National Audit of Cardiac Rehabilitation Quality and Outcomes Report 2019. 2019. Available at: https://www.bhf.org.uk/informationsupport/pu blications/statistics/national-audit-of-cardiac-rehabilitation-q uality-and-outcomes-report-2019\# (Accessed: 15 November 2021).

[18] Turk-Adawi K, Supervia M, Lopez-Jimenez F, Pesah E, Ding R, Britto RR, et al. Cardiac Rehabilitation Availability and Density around the Globe. EClinicalMedicine. 2019; 13: 31-45.

[19] Scherrenberg M, Falter M, Dendale P. Providing comprehensive cardiac rehabilitation during and after the COVID-19 pandemic. European Journal of Preventive Cardiology. 2020; 28: 520-521.

[20] Thamman R, Janardhanan R. Cardiac rehabilitation using telemedicine: the need for tele cardiac rehabilitation. Reviews in Cardiovascular Medicine. 2020; 21: 497-500.

[21] Frederix I, Vanhees L, Dendale P, Goetschalckx K. A review of telerehabilitation for cardiac patients. Journal of Telemedicine and Telecare. 2015; 21: 45-53.

[22] Knudsen MV, Petersen AK, Angel S, Hjortdal VE, Maindal HT, Laustsen S. Tele-rehabilitation and hospital-based cardiac rehabilitation are comparable in increasing patient activation and health literacy: a pilot study. European Journal of Cardiovascular Nursing. 2020; 19: 376-385.

[23] Varnfield M, Karunanithi M, Lee C, Honeyman E, Arnold D, Ding H, et al. Smartphone-based home care model improved use of cardiac rehabilitation in postmyocardial infarction patients: results from a randomised controlled trial. Heart. 2014; 100: $1770-1779$.

[24] Jin K, Khonsari S, Gallagher R, Gallagher P, Clark AM, Freedman $\mathrm{B}$, et al. Telehealth interventions for the secondary prevention of coronary heart disease: a systematic review and metaanalysis. European Journal of Cardiovascular Nursing. 2019; 18: 260-271.

[25] Anderson L, Sharp GA, Norton RJ, Dalal H, Dean SG, Jolly $\mathrm{K}$, et al. Home-based versus centre-based cardiac rehabilitation. The Cochrane Database of Systematic Reviews. 2017; 6: CD007130.

[26] Schopfer DW, Whooley MA, Allsup K, Pabst M, Shen H, Tarasovsky G, et al. Effects of Home-Based Cardiac Rehabilitation on Time to Enrollment and Functional Status in Patients with Ischemic Heart Disease. Journal of the American Heart Association. 2020; 9: e016456.

[27] Ma J, Ge C, Shi Y, Xu Y, Zhao C, Gao L, et al. Chinese HomeBased Cardiac Rehabilitation Model Delivered by Smartphone Interaction Improves Clinical Outcomes in Patients With Coronary Heart Disease. Frontiers in Cardiovascular Medicine. 2021; 8: 731557.

[28] Falter M, Scherrenberg M, Kindermans H, Kizilkilic S, Kaihara T, Dendale P. Willingness to participate in cardiac telerehabilitation: results from semi-structured interviews. European Heart Journal-Digital Health. 2021. (in press)

[29] Tang LH, Kikkenborg Berg S, Christensen J, Lawaetz J, Doherty $\mathrm{P}$, Taylor RS, et al. Patients' preference for exercise setting and its influence on the health benefits gained from exercise-based cardiac rehabilitation. International Journal of Cardiology. 2017; 232: 33-39.

[30] Scherrenberg M, Wilhelm M, Hansen D, Völler H, Cornelissen V, Frederix I, et al. The future is now: a call for action for cardiac telerehabilitation in the COVID-19 pandemic from the secondary prevention and rehabilitation section of the European Association of Preventive Cardiology. European Journal of Preventive Cardiology. 2020; 28: 524-540.

[31] Krittanawong C, Zhang H, Wang Z, Aydar M, Kitai T. Artificial Intelligence in Precision Cardiovascular Medicine. Journal of the American College of Cardiology. 2017; 69: 2657-2664.

[32] Khalaj O, Jamshidi MB, Saebnoori E, Masek B, Stadler C, Svoboda J. Hybrid Machine Learning Techniques and Computational Mechanics: Estimating the Dynamic Behavior of Oxide Precipitation Hardened Steel. IEEE Access. 2021; 9: 156930 156946.

[33] Pan Y, Zhang L. Roles of artificial intelligence in construction engineering and management: a critical review and future trends. Automation in Construction. 2021; 122: 103517.

[34] He J, Baxter SL, Xu J, Xu J, Zhou X, Zhang K. The practical implementation of artificial intelligence technologies in medicine. Nature Medicine. 2019; 25: 30-36.

[35] Topol EJ. High-performance medicine: the convergence of human and artificial intelligence. Nature Medicine. 2019; 25: 4456.

[36] Jamshidi MB, Alibeigi N, Rabbani N, Oryani B, Lalbakhsh A. Artificial Neural Networks: a Powerful Tool for Cognitive Science. 9th Annual Information Technology, Electronics and Mobile Communication Conference (IEMCON). The University of British Columbia, Vancouver, Canada. 2018.

[37] Loukis EN, Maragoudakis M, Kyriakou N. Artificial intelligence-based public sector data analytics for economic crisis policymaking. Transforming Government: People, Process and Policy. 2020; 14: 639-662.

[38] Mustaqeem, Kwon S. A CNN-Assisted Enhanced Audio Signal Processing for Speech Emotion Recognition. Sensors. 2020; 20: 183.

[39] Zhou Z, Chen K, Li X, Zhang S, Wu Y, Zhou Y, et al. Sign-tospeech translation using machine-learning-assisted stretchable sensor arrays. Nature Electronics. 2020; 3: 571-578.

[40] Rashid M, Khan MA, Alhaisoni M, Wang SH, Naqvi SR, Rehman A, et al. A Sustainable Deep Learning Framework for Object Recognition Using Multi-Layers Deep Features Fusion and Selection. Sustainability. 2020; 12: 5037.

[41] Zhang Q, Lu J, Jin Y. Artificial intelligence in recommender systems. Complex \& Intelligent Systems. 2021; 7: 439-457.

[42] Jamshidi M, Lalbakhsh A, Talla J, Peroutka Z, Hadjilooei F, Lalbakhsh P, et al. Artificial Intelligence and COVID-19: Deep Learning Approaches for Diagnosis and Treatment. IEEE Access. 2020; 8: 109581-109595.

[43] Zhou Y, Wang F, Tang J, Nussinov R, Cheng F. Artificial intelligence in COVID-19 drug repurposing. The Lancet Digital Health. 2020; 2: e667-e676.

[44] Ozturk T, Talo M, Yildirim EA, Baloglu UB, Yildirim O, Rajendra Acharya U. Automated detection of COVID-19 cases using deep neural networks with X-ray images. Computers in Biology and Medicine. 2020; 121: 103792.

[45] Jamshidi MB, Lalbakhsh A, Talla J, Peroutka Z, Roshani S, Matousek V, et al. Deep Learning Techniques and COVID-19 Drug Discovery: Fundamentals, State-of-the-Art and Future Directions. In Arpaci I, Al-Emran M, A. Al-Sharafi M, Marques G (ed.) Emerging Technologies During the Era of COVID-19 Pandemic (pp. 9-31). Cham: Springer International Publishing. 2021.

[46] Lalmuanawma S, Hussain J, Chhakchhuak L. Applications of machine learning and artificial intelligence for Covid-19 (SARS-CoV-2) pandemic: a review. Chaos, Solitons \& Fractals. 2020; 139: 110059.

[47] Hannun AY, Rajpurkar P, Haghpanahi M, Tison GH, Bourn C, Turakhia MP, et al. Cardiologist-level arrhythmia detection and classification in ambulatory electrocardiograms using a deep neural network. Nature Medicine. 2019; 25: 65-69.

[48] Wu C, Hsu W, Islam MM, Poly TN, Yang H, Nguyen PA, et $a l$. An artificial intelligence approach to early predict non-STelevation myocardial infarction patients with chest pain. Computer Methods and Programs in Biomedicine. 2019; 173: 109- 
117.

[49] Dawes TJW, de Marvao A, Shi W, Fletcher T, Watson GMJ, Wharton $\mathrm{J}$, et al. Machine Learning of Three-dimensional Right Ventricular Motion Enables Outcome Prediction in Pulmonary Hypertension: a Cardiac MR Imaging Study. Radiology. 2017; 283: 381-390.

[50] Thijs I, Fresiello L, Oosterlinck W, Sinnaeve P, Rega F. Assessment of Physical Activity by Wearable Technology During Rehabilitation After Cardiac Surgery: Explorative Prospective Monocentric Observational Cohort Study. JMIR mHealth and uHealth. 2019; 7: e9865.

[51] Binkley PF, Frontera W, Standaert DG, Stein J. Predicting the potential of wearable technology - Physicians share their vision of future clinical applications of wearable technology. IEEE Engineering in Medicine and Biology Magazine. 2003; 22: 23-27.

[52] De Cannière H, Corradi F, Smeets CJP, Schoutteten M, Varon C, Van Hoof $\mathrm{C}$, et al. Wearable Monitoring and Interpretable Machine Learning Can Objectively Track Progression in Patients during Cardiac Rehabilitation. Sensors. 2020; 20: 3601.

[53] Munos B, Baker PC, Bot BM, Crouthamel M, de Vries G, Ferguson I, et al. Mobile health: the power of wearables, sensors, and apps to transform clinical trials. Annals of the New York Academy of Sciences. 2016; 1375: 3-18.

[54] Zheng Q, Tang Q, Wang ZL, Li Z. Self-powered cardiovascular electronic devices and systems. Nature Reviews Cardiology. 2021; 18: 7-21.

[55] Chiauzzi E, Rodarte C, DasMahapatra P. Patient-centered activity monitoring in the self-management of chronic health conditions. BMC Medicine. 2015; 13: 77.

[56] Vooijs M, Alpay LL, Snoeck-Stroband JB, Beerthuizen T, Siemonsma PC, Abbink JJ, et al. Validity and usability of lowcost accelerometers for internet-based self-monitoring of physical activity in patients with chronic obstructive pulmonary disease. Interactive Journal of Medical Research. 2014; 3: e14.

[57] Shameer K, Johnson KW, Glicksberg BS, Dudley JT, Sengupta PP. Machine learning in cardiovascular medicine: are we there yet? Heart. 2018; 104: 1156-1164.

[58] Dias D, Cunha JPS. Wearable Health Devices-Vital Sign Monitoring, Systems and Technologies. Sensors. 2018; 18: 2414.

[59] Chaari M, Abid M, Ouakrim Y, Lahami M, Mezghani N. A Mobile Application for Physical Activity Recognition using Acceleration Data from Wearable Sensors for Cardiac Rehabilitation. 13th International Joint Conference on Biomedical Engineering Systems and Technologies. Valletta, Malta. 2020.

[60] Pervaiz U, Khawaldeh S, Aleef TA, Minh VH, Hagos YB. Activity monitoring and meal tracking for cardiac rehabilitation patients. International Journal of Medical Engineering and Informatics. 2018; 10: 252.

[61] Prabhu G, O’Connor NE, Moran K. Recognition and Repetition Counting for Local Muscular Endurance Exercises in ExerciseBased Rehabilitation: A Comparative Study Using Artificial Intelligence Models. Sensors. 2020; 20: 4791.

[62] Dor-Haim H, Katzburg S, Leibowitz D. A Novel Digital Platform for a Monitored Home-based Cardiac Rehabilitation Program. Jove-Journal of Visualized Experiments. 2019.

[63] Rasekaba T, Lee AL, Naughton MT, Williams TJ, Holland AE. The six-minute walk test: a useful metric for the cardiopulmonary patient. Internal Medicine Journal. 2009; 39: 495-501.

[64] Salvi D, Poffley E, Orchard E, Tarassenko L. The Mobile-Based 6-Minute Walk Test: Usability Study and Algorithm Development and Validation. JMIR mHealth and uHealth. 2020; 8: e13756.

[65] Sana F, Isselbacher EM, Singh JP, Heist EK, Pathik B, Armoundas AA. Wearable Devices for Ambulatory Cardiac Monitoring: JACC State-of-the-Art Review. Journal of the American College of Cardiology. 2020; 75: 1582-1592.
[66] Doimo S, Fabris E, Piepoli M, Barbati G, Antonini-Canterin F, Bernardi G, et al. Impact of ambulatory cardiac rehabilitation on cardiovascular outcomes: a long-term follow-up study. European Heart Journal. 2018; 40: 678-685.

[67] Batalik L, Dosbaba F, Hartman M, Konecny V, Batalikova K, Spinar J. Long-term exercise effects after cardiac telerehabilitation in patients with coronary artery disease: 1-year follow-up results of the randomized study. European Journal of Physical and Rehabilitation Medicine. 2021; 57: 807-814.

[68] Frederix I, Solmi F, Piepoli MF, Dendale P. Cardiac telerehabilitation: a novel cost-efficient care delivery strategy that can induce long-term health benefits. European Journal of Preventive Cardiology. 2017; 24: 1708-1717.

[69] Laustsen S, Oestergaard LG, van Tulder M, Hjortdal VE, Petersen AK. Telemonitored exercise-based cardiac rehabilitation improves physical capacity and health-related quality of life. Journal of Telemedicine and Telecare. 2020; 26: 36-44.

[70] Thomas RJ, Beatty AL, Beckie TM, Brewer LC, Brown TM, Forman DE, et al. Home-Based Cardiac Rehabilitation: a Scientific Statement from the American Association of Cardiovascular and Pulmonary Rehabilitation, the American Heart Association, and the American College of Cardiology. Circulation. 2019; 140: E69-E89.

[71] Keteyian SJ, Grimshaw C, Brawner CA, Kerrigan DJ, Reasons L, Berry R, et al. A Comparison of Exercise Intensity in Hybrid Versus Standard Phase Two Cardiac Rehabilitation. Journal of Cardiopulmonary Rehabilitation and Prevention. 2021; 41: 1922.

[72] Ledger D, McCaffrey D. Inside wearables: How the science of human behavior change offers the secret to long-Term engagement. Endeavour Partners, LLC. 2014; 93: 36-45.

[73] Wongvibulsin S, Habeos EE, Huynh PP, Xun H, Shan RZ, Rodriguez KAP, et al. Digital Health Interventions for Cardiac Rehabilitation: Systematic Literature Review. Journal of medical Internet research. 2021; 23: e18773.

[74] World Health Organization. Coronavirus disease (COVID19) Situation Report-194. 2020. Available at: https: //www.who.int/docs/default-source/coronaviruse/situation-rep orts/20200801- covid-19-sitrep-194.pdf?sfvrsn=401287f3 _ 2 (Accessed: 20 November 2021).

[75] Neubeck L, Redfern J, Fernandez R, Briffa T, Bauman A, Freedman SB. Telehealth interventions for the secondary prevention of coronary heart disease: a systematic review. European Journal of Cardiovascular Prevention and Rehabilitation. 2009; 16: 281-289.

[76] Vale MJ, Jelinek MV, Best JD, Dart AM, Grigg LE, Hare DL, et al. Coaching patients on Achieving Cardiovascular Health $(\mathrm{COACH})$ : a multicenter randomized trial in patients with coronary heart disease. Archives of Internal Medicine. 2003; 163: 2775-2783.

[77] Mittag O, China C, Hoberg E, Juers E, Kolenda K, Richardt G, et al. Outcomes of cardiac rehabilitation with versus without a follow-up intervention rendered by telephone (Luebeck followup trial): overall and gender-specific effects. International Journal of Rehabilitation Research.. 2007; 29: 295-302.

[78] Neubeck L, Lowres N, Benjamin EJ, Freedman SB, Coorey G, Redfern J. The mobile revolution-using smartphone apps to prevent cardiovascular disease. Nature Reviews Cardiology. 2015; 12: $350-360$.

[79] Chow CK, Redfern J, Hillis GS, Thakkar J, Santo K, Hackett ML, et al. Effect of Lifestyle-Focused Text Messaging on Risk Factor Modification in Patients with Coronary Heart Disease. The Journal of the American Medical Association. 2015; 314: 1255.

[80] Pandey A, Krumme AA, Patel T, Choudhry NK. The Impact of Text Messaging on Medication Adherence and Exercise Among 
Postmyocardial Infarction Patients: Randomized Controlled Pilot Trial. JMIR mHealth and uHealth. 2017; 5: e110.

[81] Zutz A, Ignaszewski A, Bates J, Lear SA. Utilization of the Internet to Deliver Cardiac Rehabilitation at a Distance: a Pilot Study. Telemedicine and E-Health. 2007; 13: 323-330.

[82] Southard BH, Southard DR, Nuckolls J. Clinical Trial of an Internet-based Case Management System for Secondary Prevention of Heart Disease. Journal of Cardiopulmonary Rehabilitation. 2003; 23: 341-348.

[83] Kikuchi A, Taniguchi T, Nakamoto K, Sera F, Ohtani T, Yamada $\mathrm{T}$, et al. Feasibility of home-based cardiac rehabilitation using an integrated telerehabilitation platform in elderly patients with heart failure: a pilot study. Journal of Cardiology. 2021; 78: 6671.

[84] Dorje T, Zhao G, Tso K, Wang J, Chen Y, Tsokey L, et al. Smartphone and social media-based cardiac rehabilitation and secondary prevention in China (SMART-CR/SP): a parallel-group, single-blind, randomised controlled trial. The Lancet Digital Health. 2019; 1: e363-e374.

[85] Widmer RJ, Allison TG, Lennon R, Lopez-Jimenez F, Lerman LO, Lerman A. Digital health intervention during cardiac rehabilitation: a randomized controlled trial. American Heart Journal. 2017; 188: 65-72.

[86] Tripoliti EE, Karanasiou GS, Kalatzis FG, Bechlioulis A, Goletsis Y, Naka K, et al. HEARTEN KMS - a knowledge management system targeting the management of patients with heart failure. Journal of Biomedical Informatics. 2019; 94: 103203.

[87] Lowres N, Duckworth A, Redfern J, Thiagalingam A, Chow CK. Use of a Machine Learning Program to Correctly Triage Incoming Text Messaging Replies From a Cardiovascular TextBased Secondary Prevention Program: Feasibility Study. JMIR mHealth and uHealth. 2020; 8: e19200.

[88] Falter M, Scherrenberg M, Dendale P. Digital Health in Cardiac Rehabilitation and Secondary Prevention: A Search for the Ideal Tool. Sensors. 2020; 21: 12.

[89] Gevaert AB, Adams V, Bahls M, Bowen TS, Cornelissen V, Dörr $\mathrm{M}$, et al. Towards a personalised approach in exercisebased cardiovascular rehabilitation: how can translational research help? A 'call to action' from the Section on Secondary Prevention and Cardiac Rehabilitation of the European Association of Preventive Cardiology. European Journal of Preventive Cardiology. 2020; 27: 1369-1385.

[90] Haro Alonso D, Wernick MN, Yang Y, Germano G, Berman DS, Slomka P. Prediction of cardiac death after adenosine myocardial perfusion SPECT based on machine learning. Journal of $\mathrm{Nu}-$ clear Cardiology. 2019; 26: 1746-1754.

[91] Young L, Zhang Q, Lian E, Roberts K, Weintraub N, Dong Y, et al. Factors Predicting the Utilization of Center-Based Cardiac Rehabilitation Program. Geriatrics. 2020; 5: 66.

[92] Schmied C. 'Cardiac rehabilitation works': but it should be tailored individually, started early, and followed for a lifetime. European Heart Journal. 2018; 40: 686-688.

[93] Smith B, Magnani JW. New technologies, new disparities: the intersection of electronic health and digital health literacy. International Journal of Cardiology. 2019; 292: 280-282.

[94] Makridakis S. The forthcoming Artificial Intelligence (AI) rev- olution: its impact on society and firms. Futures. 2017; 90: 4660.

[95] Olhede SC, Wolfe PJ. The growing ubiquity of algorithms in society: implications, impacts and innovations. Philosophical Transactions of the Royal Society a: Mathematical, Physical and Engineering Sciences. 2018; 376: 20170364.

[96] Jobin A, Ienca M, Vayena E. The global landscape of AI ethics guidelines. Nature Machine Intelligence. 2019; 1: 389-399.

[97] Visco V, Ferruzzi GJ, Nicastro F, Virtuoso N, Carrizzo A, Galasso G, et al. Artificial Intelligence as a Business Partner in Cardiovascular Precision Medicine: an Emerging Approach for Disease Detection and Treatment Optimization. Current Medicinal Chemistry. 2021; 28: 6569-6590.

[98] Dorado-Díaz PI, Sampedro-Gómez J, Vicente-Palacios V, Sánchez PL. Applications of Artificial Intelligence in Cardiology. the Future is already here. Revista EspañOla De Cardiología. 2019; 72: 1065-1075.

[99] McCall B. What does the GDPR mean for the medical community? The Lancet. 2018; 391: 1249-1250.

[100] Vayena E, Blasimme A, Cohen IG. Machine learning in medicine: Addressing ethical challenges. PLoS Medicine. 2018; 15: e1002689.

[101] Cho MK. Rising to the challenge of bias in health care AI. Nature Medicine. 2021; 27: 2079-2081.

[102] Reddy S, Allan S, Coghlan S, Cooper P. A governance model for the application of AI in health care. Journal of the American Medical Informatics Association. 2020; 27: 491-497.

[103] Char DS, Shah NH, Magnus D. Implementing Machine Learning in Health Care - Addressing Ethical Challenges. New England Journal of Medicine. 2018; 378: 981-983.

[104] Char DS, Abràmoff MD, Feudtner C. Identifying Ethical Considerations for Machine Learning Healthcare Applications. The American Journal of Bioethics. 2020; 20: 7-17.

[105] Lepri B, Oliver N, Letouzé E, Pentland A, Vinck P. Fair, Transparent, and Accountable Algorithmic Decision-making Processes. Philosophy \& Technology. 2018; 31: 611-627.

[106] Asan O, Bayrak AE, Choudhury A. Artificial Intelligence and Human Trust in Healthcare: Focus on Clinicians. Journal of Medical Internet Research. 2020; 22: e15154.

[107] Tran V, Riveros C, Ravaud P. Patients' views of wearable devices and $\mathrm{AI}$ in healthcare: findings from the ComPaRe ecohort. NPJ Digital Medicine. 2019; 2: 53.

[108] Duncker D, Ding WY, Etheridge S, Noseworth PA, Veltmann C, Yao XX, et al. Smart Wearables for Cardiac Monitoring-RealWorld Use beyond Atrial Fibrillation. Sensors. 2021; 21: 2539.

[109] Shah H. Algorithmic accountability. Philosophical Transactions. Series a, Mathematical, Physical, and Engineering Sciences. 2018; 376: 20170362

[110] Howard A. Are We Trusting AI Too Much? Examining Human-Robot Interactions in the Real World. In Proceedings of the $2020 \mathrm{ACM} /$ IEEE International Conference on Human-Robot Interaction. Cambridge, United Kingdom: Association for Computing Machinery. 2020.

[111] Shneiderman B. Human-Centered Artificial Intelligence: Reliable, Safe \& Trustworthy. International Journal of HumanComputer Interaction. 2020; 36: 495-504. 